\title{
Development of a model for membrane filtration of long and flexible macromolecules: Application to predict dextran and linear DNA rejections in ultrafiltration
}

\author{
António Morão ${ }^{\mathrm{a}, \mathrm{e}, *}$, José C. Nunes ${ }^{\mathrm{e}}$, Fani Sousa ${ }^{\mathrm{e}}$, Maria Teresa Pessoa de Amorim ${ }^{\mathrm{b}}$, \\ Isabel C. Escobar ${ }^{\text {, }}$ João A. Queiroz ${ }^{\text {a,c,e }}$ \\ a Unidade de Materiais Têxteis e Papeleiros, University of Beira Interior, 6201-001 Covilhã, Portugal \\ ${ }^{\mathrm{b}}$ Department of Textile Engineering, University of Minho, 4800-058 Guimarães, Portugal \\ ${ }^{\mathrm{c}}$ Department of Chemistry, University of Beira Interior, 6201-001 Covilhã, Portugal \\ d Department of Chemical and Environmental Engineering, University of Toledo, Toledo, $\mathrm{OH} 43606$, United States \\ e Centro de Investigação em Ciências da Saúde, University of Beira Interior, 6201-001 Covilhã, Portugal
}

\section{A R T I C L E I N F O}

\section{Article history:}

Received 21 October 2008

Received in revised form 6 March 2009

Accepted 6 March 2009

Available online 20 March 2009

\section{Keywords:}

Microfiltration

Ultrafiltration

DNA

Dextran

Modeling

\begin{abstract}
A B S T R A C T
A model is proposed for predicting the intrinsic rejections of linear flexible molecules, of high molecular dimensions, in microfiltration and ultrafiltration membranes. The developed model is based on the available theory of hindered transport of solutes through narrow pores, assuming that convection is the only relevant transport mechanism. For the application of the model, partition coefficients of the solutes between the solution and the membrane were estimated by a stochastic simulation, assuming suction of the macrosolutes into the pore. In these simulations, the macromolecules were modeled as freely jointed chains. Different methods for the estimation of the molecular dimensions of the solutes are discussed. The validity of the model was tested using dextran T2000 and plasmid pUC19 in its linear isoform, as model solutes; the tests were performed using membranes of different pore size, that include two ultrafiltration membranes previously characterized, having 4.1 and $10.5 \mathrm{~nm}$ of pore radius, and two track-etched polycarbonate membranes, having 15 and $40 \mathrm{~nm}$ of pore radius. Filtration was performed in a stirred cell, at controlled permeate flux. Different conditions of stirring speed and permeate flux were tested to check the consistency of the model. The results show that very satisfactory predictions are obtained, considering the simplicity of the model, especially in the case of the plasmid.
\end{abstract}

(c) 2009 Elsevier B.V. All rights reserved.

\section{Introduction}

The development of hindered transport models that describe the permeation of solutes through porous matrices is of central importance in membrane characterization, and prediction of selectivities. Models in which the solutes are assumed to behave like hard spheres (HS), have been widely used for this purpose, with remarkable success in microfiltration, ultrafiltration and nanofiltration. The main advantage of this assumption is that, in the case of cylindrical pores and slits, it enables the calculation of hydrodynamic parameters related with hindered diffusive and convective transport inside the membranes [1-3]. If charge effects are absent, i.e., if the solutes are neutral or there is a high concentration of salts, this is usually enough to obtain satisfactory predictions of solute intrinsic

\footnotetext{
* Corresponding author at: Unidade de Materiais Têxteis e Papeleiros, University of Beira Interior, Rua Marquês d'Ávila e Bolama, 6201-001 Covilhã, Portugal. Tel.: +351275322304.

E-mail address: ammorao@ubi.pt (A. Morão).
}

rejections, only based on size exclusion/steric effects. Consequently, this approach has been widely adopted for obtaining membrane structural parameters, namely the pore size or pore size distribution [2,4-6] and, more recently information about membrane asymmetry [7]. After membrane characterization, the HS approach enables the prediction of solute rejections from the knowledge of their hydrodynamic radii, and therefore the membrane selectivity; this information is of the utmost importance when selecting membranes for a particular application, for interpretation of experimental results of laboratory tests and also for process optimization.

However, in the application of these models to study the permeation of very long linear molecules of high molecular weight, significant deviations from hard sphere behavior have been found in practice. In fact, high permeation of these molecules is observed in membranes with pore radii much smaller than the molecular dimensions of the solutes, often expressed as the hydrodynamic radius, $r_{s}$, or the radius of gyration, $r_{g}$. It should be noted that in the case of flexible molecules both these properties should be viewed as average quantities. A recent review on the subject can be found elsewhere [8]. This result has the clear physical meaning that for 
flexible molecules of very high molecular weight, especially above $\sim 1 \mathrm{MDa}$, and $r_{g}$ in the order of $10 \mathrm{~nm}$, the approach of hard spheres is clearly unrealistic; therefore, it becomes necessary to develop more adequate models, that include the possibility of the molecule to adopt different conformations along the time, and, if necessary, the effects of molecular deformation induced by the convective flow of the solvent through the membrane.

In the conventional approach for modeling the retention of flexible molecules, three different situations can be considered: the permeation in the absence of convection (transport exclusively done by diffusion), the permeation at moderate fluxes without molecular deformation and the permeation at high fluxes, where molecular deformation can be high [1]. Among the different published studies in this area, it is also important to distinguish those in that the ratio between the solute radius to the pore radius $\left(\lambda_{s}\right)$ is less than 1 , from those cases in that $\lambda_{S}>1$. In the case of linear molecules, for $\lambda_{s}$ values up to 0.87 theoretical hydrodynamic models are available for prediction of hindered transport of flexible linear macromolecules [9]. However, for $\lambda_{s}$ values greater than 0.87 , the existing attempts of modeling solute transport in membrane filtration are much difficult, especially for $\lambda_{s}>1$, since molecular deformation has to be considered.

Besides the transport model, the estimation of partition coefficients at the pore entrance is also necessary. For this purpose, stochastic methods, involving computational simulation, have been developed [10-12] in order to avoid the excessive complexity of obtaining an analytical solution. In these algorithms it is estimated the probability of a certain event to occur, for instance, the probability of a molecule to enter inside a pore, by testing that occurrence several times (typically more than $10^{5}$ times are required to obtain a satisfactory approach to actual probability of the event). The way in which the occurrence is tested can be diverse, depending on the applied method, and of course the way in which the event is described can be done with a higher or lower degree of realism, with important consequences in terms of computational effort. To avoid excessive computational power requirements for simulating the structure of flexible molecules, molecular prototypes that mimic the structure of the molecules studied, and retain the essential characteristics that determine the permeation through membrane pores, can be defined.

The simplest prototype that can be considered for modeling a linear macromolecule is that of an equivalent statistical freely jointed chain (FJC) [13-15]. This model, and other more sophisticated models, have been used for studying the possibility of the macromolecules to adopt conformations and enter into confined regions, namely pores and slits [10-12], yet never in the case of $\lambda_{s} \gg 1$. Predictions of partition coefficients can be made by determining the probability of the molecule to adopt such conformations, if one assumes no deformation induced by the convective flow through the pores. However, in pressure driven membrane processes, partition coefficients should not be estimated using this approach, and it can be shown that calculated rejections using these estimates are not in accordance with the reality. In fact, in the field of membrane applications, these estimations have focused mainly the study of hindered diffusion phenomena. In the present work it is considered that partition coefficients are influenced by the convective flow, being suction and the molecule spatial orientation considered the essential factors that determine partition.

Thus, it was the aim of this work to develop a simple model, based on previous models of hindered molecular transport in porous membranes, and estimation of partition coefficients, in the case of $\lambda_{s} \gg 1$. This model can be generally applied for the prediction of membrane rejections of linear macromolecules, represented as FJCs. Its validity was checked by studying the permeation of two linear and long molecules through different membranes. The solutes tested were dextran T2000 (which has a molecular weight near $2 \mathrm{MDa}$ ) and plasmid pUC19 (which has 2686 base pairs, and $1.773 \mathrm{MDa}$ of molecular weight) treated with a restriction enzyme, that cuts the double stranded DNA chain, resulting in a linear isoform.

\section{Development of the model}

\subsection{Definition of the molecular prototypes}

A FJC can be defined as a series of straight segments, $n_{k}$, with random orientations and all with the same length, $l_{k}$, connecting a number of dot masses [13-15]. The values of $n_{k}$ and $l_{k}$ are defined in order to obtain the same contour length of the real molecule, $L ; l_{k}$ corresponds to the minimum distance for having each segment with an orientation independent from that of the adjacent segments, and is called Kuhn distance. Therefore, the ratio $l_{k} / L$ reflects the stiffness of the chain. Such a structure can be computationally generated, as a random-walk of $n_{k}$ steps in three dimensions, in which each step has a length $l_{k}$ [15]. Mathematically such a random-walk process is called Markoffian, since it represents the evolution of a memoryless system (the position in space after each step is independent from the previous positions); any kind of interactions between the monomers are therefore neglected, and a FJC, defined in this way, is also called an ideal chain. As it can be seen, the FJC model is the simplest model that can be considered for representing a flexible linear molecule, being particularly adequate for representing long molecules, and especially when $l_{k} / L$ is small. If one considers the computation effort required to simulate very detailed molecular structures, and the need to simulate the permeation event a high number of times (in order to obtain significant estimates of the probability of permeation), the FJC model was the natural choice, in a first approach, for the setting-up of molecular prototypes of the molecules studied in this work.

For a FJC the average end-to-end distance of the chain, $\left\langle h^{2}\right\rangle^{1 / 2}$, is the simplest way to define its dimensions, and it can be shown that is simply given by $n_{k}^{1 / 2} l_{k}$, independently of the number of segments $[14,15]$. The value of $\left\langle h^{2}\right\rangle^{1 / 2}$ is related with the radius of gyration of the molecule, $r_{g}$, which can be experimentally determined. For a molecule, having $N$ atoms, each one having a mass $m_{i}$, and positioned at a distance $r_{i}(i=0,1, \ldots, N-1)$ from the center of mass of the molecule the instantaneous radius of gyration $r_{g}^{*}$ is given by:

$r_{g}^{*}=\sqrt{\frac{\sum_{i=0}^{N-1} m_{i} r_{i}^{2}}{\sum_{i=0}^{N-1} m_{i}}}$

In the case of a statistically equivalent FJC, representing a polymer, the various atomic masses are replaced by $n_{k}$ equal dot masses, and since the molecule is continuously changing its conformation, it is the mean radius of gyration, taken for a high number of possible conformations, the quantity related to the experimental values of $r_{g}$. That average is given by:

$r_{g}=\left\langle r_{g}^{*}\right\rangle=\sqrt{\frac{\sum_{i=0}^{n_{k}}\left\langle\left(r_{i}-r_{c m}\right)^{2}\right\rangle}{n_{k}+1}}=\sqrt{\frac{\sum_{i=0}^{n_{k}} \sum_{j=0}^{n_{k}}\left\langle\left(r_{i}-r_{j}\right)^{2}\right\rangle}{2\left(n_{k}+1\right)^{2}}}$

where $r_{c m}$ are the coordinates of the center of mass. Independently of the number of segments of the FJC, the average radius of gyration, $r_{g}$ is given by [15]:

$r_{g}=l_{k} \sqrt{\frac{n_{k}\left(n_{k}+2\right)}{6\left(n_{k}+1\right)}}$ 
In the limiting case of a large number of segments, $n_{k}$, the FJC is called a Gaussian chain and one obtains $[14,15]$ :

$r_{g}=\frac{n_{k}^{1 / 2} l_{k}}{\sqrt{6}}=\frac{\left\langle h^{2}\right\rangle^{1 / 2}}{\sqrt{6}}$

\subsection{Estimation of the dimension of the real chains}

For specifying the dimensions of the prototypes, firstly it is necessary to know the dimensions of the real chains. Although end-to-end distances have been determined by AFM image analysis [16] this information is relatively scarce, and experimental data on gyration radii, obtained by light scattering techniques, or from other experimental quantities that can be related with $r_{g}$, namely diffusion coefficients and values of persistence length, provide the main source of information. For these estimations, some theoretical improvements of the FJC model have to be considered. References [15,17] present a detailed review of most important theoretical developments in this area of molecular modeling. It should be pointed out that, the estimation of the relationship between $r_{g}$ and the hydrodynamic radius $\left(r_{s}\right)$, is one of the main objectives of these theories. Therefore, $r_{g}$ values may be estimated from hydrodynamic radii or diffusion coefficients, $D_{\infty}$, with these being related each other by the Stokes-Einstein equation:

$D_{\infty}=\frac{k_{B} T}{6 \pi \eta r_{s}}$

where $k_{B}$ is the Boltzmann constant, $T$ is the absolute temperature and $\eta$ is the solvent viscosity. Experimental values of $D_{\infty}$, determined by different techniques, including light scattering, sedimentation and Brownian motion tracking of single molecules, are often available in literature. In the case of a Gaussian chain, in a theta solvent (which is a solvent where the excluded volume effects due to the dimensions of the monomers are exactly cancelled by the interaction of the polymer chain with the solvent), the radius of gyration can be calculated from the hydrodynamic radius, according to Zimm model $[15,18,19]$ :

$D_{\infty}=\frac{8}{3 \sqrt{6 \pi^{3}}} \frac{k_{B} T}{\eta l_{k} n_{k}^{1 / 2}} \Rightarrow \frac{r_{g}}{r_{s}}=\frac{8}{3 \sqrt{\pi}}=1.505$

In a good solvent, it is estimated, from the renormalization group theory, that the relationship between $r_{g}$ and $r_{s}$ is the following $[15,20]$ :

$D_{\infty}=0.0829 \frac{k_{B} T}{\eta r_{g}} \Rightarrow \frac{r_{g}}{r_{s}}=6 \pi(0.0829)=1.563$

As it can be seen, the difference between the values of the ratio $r_{g} / r_{s}$ calculated by equations (6) and ( 7$)$ is small $(<5 \%)$, and therefore, both equations can be considered for an estimate of $r_{g}$. However, it should be remarked that these equations are only valid for Gaussian chains. When the number of segments is small, or more specifically when the ratio of the Kuhn distance to the contour length, $l_{k} / L$, is not small enough to consider the chain as Gaussian, the so-called worm-like chain model $[15,17]$ is preferable for estimating chain dimensions. A "persistent" or "worm-like" (WLC) chain is a spatial line of continuous curvature, thus, unlike the FJC, which consists of discrete segments. The measure of equilibrium rigidity of such a chain is its persistence length, " $a$ ". When the contour length equals the persistence length a WLC is similar to a poorly bent rod, but when $L$ is large enough the chain has the conformation of a Gaussian coil, for which the Kuhn length is $l_{k}=2 a[13,15,21]$. The WLC model was originally proposed by Kratky and Porod [22]. The average endto-end distance, and the radius of gyration of a WLC are simple functions of the contour length of the polymer and the persistence

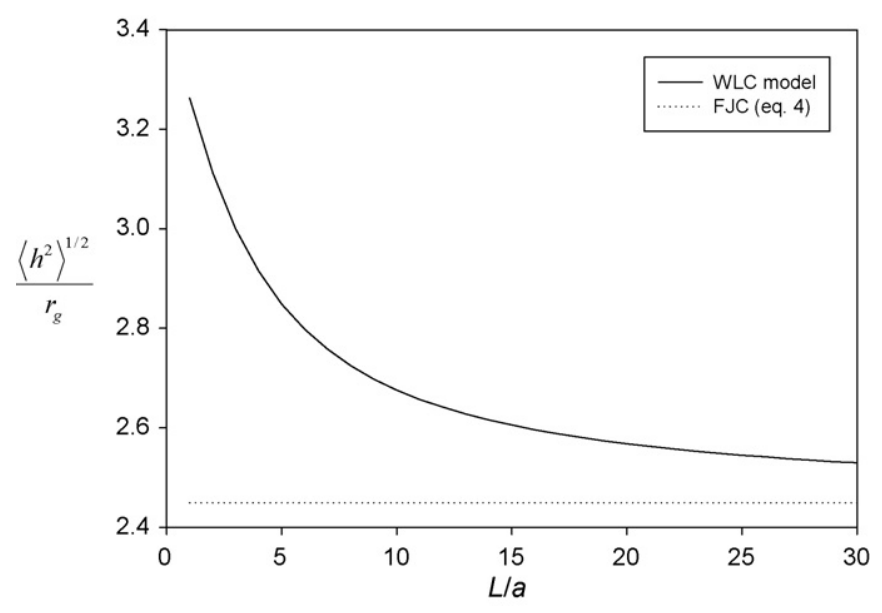

Fig. 1. Theoretical dependence of $\left(\left\langle h^{2}\right\rangle^{1 / 2} / r_{g}\right)$ ratio on $L / a$, for a worm-like chain (WLC) and comparison with the freely jointed chain (FJC) model.

length [15]:

$\left\langle h^{2}\right\rangle^{1 / 2}=(2 a)^{1 / 2}\left[L+a\left(\mathrm{e}^{-L / a}-1\right)\right]^{1 / 2}$

and

$r_{g}=a\left[\frac{L}{3 a}-1+\frac{2 a}{L}-2\left(\frac{a}{L}\right)^{2}\left(1-\mathrm{e}^{-L / a}\right)\right]^{1 / 2}$

The dependence of $\left(\left\langle h^{2}\right\rangle^{1 / 2} / r_{g}\right)$ ratio on $L / a$ is shown in Fig. 1 . As it can be seen, about $L / a=10$ the difference between that ratio and the asymptotic value given by equation (4) is only of $\sim 10 \%$. Therefore, one may conclude that there is a clear similitude between the WLC and FJC representations of a macromolecule for $L / a$ higher than $\sim 10$. Although the persistence length values that commonly appear in literature are obtained from experimental values of $r_{g}$, it can be independently estimated by force-extension measurements [16,23].

\subsection{Specification of the molecular size of the prototypes}

For the type of molecules studied in this work, dextrans and linear double stranded DNA, the main source of information from which the molecular dimensions can be estimated are diffusion coefficient values (or $r_{s}$, which are intimately related quantities, as previous seen).For dextrans, the following relationship was used for the estimation of $r_{s}$, as a function of the molecular weight, $M_{w}$ [4]:

$r_{s}=0.0282\left(M_{w}\right)^{0.47752}$

with $r_{s}$ in nm and $M_{w}$ in Da. Thus, for dextran T2000, since it has a normative molecular weight of $2 \times 10^{6} \mathrm{Da}$, it can be estimated that $r_{s}=28.8 \mathrm{~nm}, r_{g}=45 \mathrm{~nm}$ (using equation (7)) and $\left\langle h^{2}\right\rangle^{1 / 2}=110 \mathrm{~nm}$ (using equation (4)).

In the case of linear DNA, the dependence of $D_{\infty}$ on the number of base pairs is analyzed in Fig. 2a. Two main techniques provide the data available in literature, light scattering (LS) techniques [40-44] and Brownian motion tracking (BMT) of fluorescently labeled molecules $[19,24,45,46]$. The experimental values are not equivalent, as pointed out by Smith et al. [24], and a factor of $(1.75)^{2 / 5}$ was proposed by these authors, to correct the BMT values. In fact, as it may be seen in Fig. 2a, the values obtained by BMT have a clear tendency to be lower than those obtained by LS, and this is likely to be due to the binding of the dye used in these determinations, which intercalates in the DNA chain [24]. Using LS and 

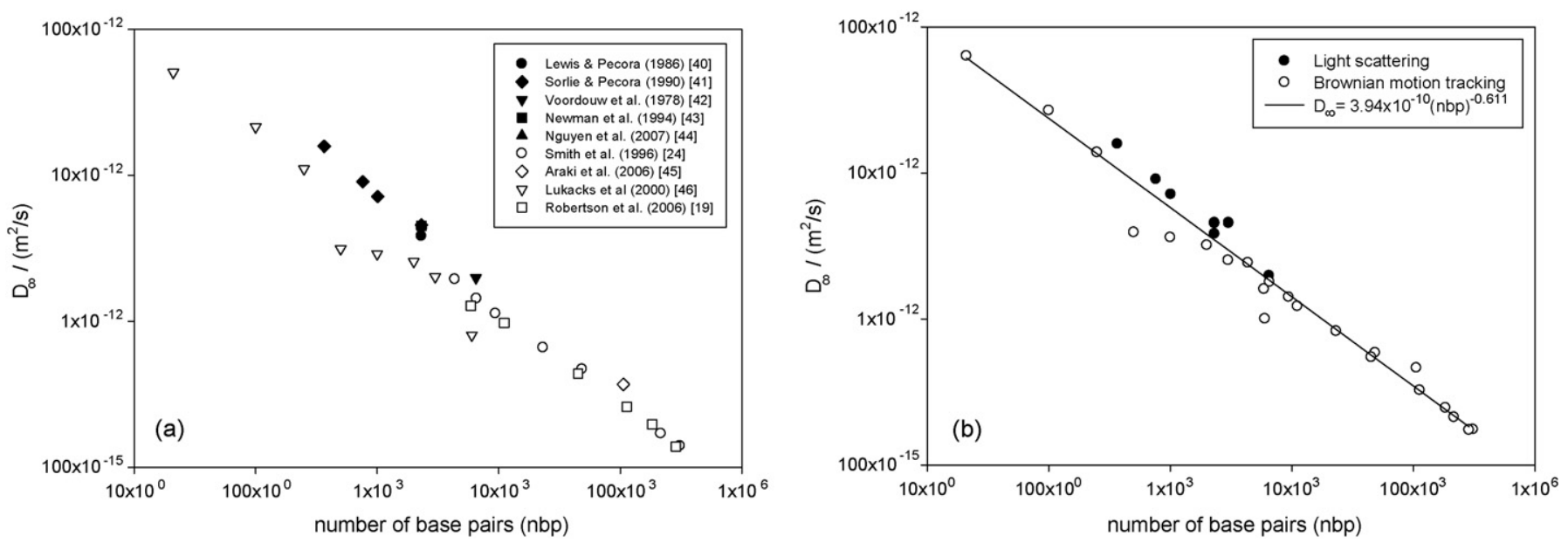

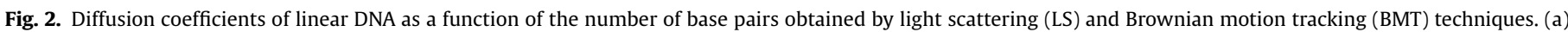
Values as found in literature (white-BMT; black-LS); (b) plot of equation (11), LS values (black) and BMT corrected values (white).

corrected BMT values, the following relationship can be obtained from the fit shown in Fig. 2b:

$D_{\infty}=3.94 \times 10^{-10}(n b p)^{-0.611}$

Then using the Stokes-Einstein equation, one gets:

$r_{s}=6.27 \times 10^{-10}(n b p)^{0.611}$

Since for the plasmid pUC19, $n b p=2686$, therefore $r_{s}=77.9 \mathrm{~nm}$, $r_{g}=122 \mathrm{~nm}$ and $\left\langle h^{2}\right\rangle^{1 / 2}=298 \mathrm{~nm}$. These values can be compared with those estimated by a different approach, using the WLC model, which is often applied for modeling DNA (since DNA is a molecule of considerable stiffness). This implies the knowledge of the contour and persistence lengths of the polymer. The persistence length of double stranded linear DNA is not a constant; on the contrary, it varies considerably with ionic strength. Two broad regions can be distinguished [21]: for values up to $10 \mathrm{mM}$, there is a rapid decrease with the increase in the ionic strength $\left(\mathrm{Na}^{+}\right.$solutions) with values of $80-100 \mathrm{~nm}$ being reported in this region; however, above $10 \mathrm{mM}$ the rate of increase slows down, and values between 45 and $50 \mathrm{~nm}$ are usually found in a considerable range for ionic strength $[8,21,23,25,26]$. This effect may be related with the intramolecular repulsion of the charged phosphate groups of the DNA chain. In accordance to this possibility is the fact that $\mathrm{Mg}^{2+}$ ions have a significantly higher effect on the decrease of the persistence length of DNA [21] and also the recently obtained results of the study of short double stranded DNA oligomers, that show persistence length values as low as $9 \mathrm{~nm}$ [27]. About the contour length, this can be readily estimated from the value of the height of the helix of double stranded B-DNA, per each pair of bases, $0.34 \mathrm{~nm}$ [28] times the number of base pairs, which gives $913 \mathrm{~nm}$, since the plasmid pUC19 has 2686 base pairs (bp). Thus, using the approximate value of $a=50 \mathrm{~nm}$ it is obtained using equations (8) and (9) that $r_{g}=114 \mathrm{~nm}$ and $\left\langle h^{2}\right\rangle^{1 / 2}=294 \mathrm{~nm}$ for linear pUC19. These values are in agreement with those obtained using the FJC model, previously calculated.

\subsection{Partition coefficients}

The next step in the set-up of the model is the estimation of steric partition coefficients at the pore entrance. To simulate the partition, a Monte Carlo method based on that of Davidson et al. [10] was developed here. That previous method consists in the determination of the radial distribution of the probability of the molecule (prototype) to enter inside the pore, with the distribution being obtained from stochastic simulations, at 10 different radial positions, plus the center of the pore. Thus, after the molecule is generated, the center of mass is placed in a certain radial position, and it is tested if the coordinates of the projection of all points (dot masses in the prototypes) on the plane of the membrane surface, are inside the pore; only if that is the case, a "success" is recorded. Here, the testing procedure is changed by taking into account the high flexibility of the molecules tested, and by considering the possibility of molecular deformation due to suction at the pore entrance. Therefore, after generation the 3D structure of the molecule and aligning its center of mass with the center of the pore, the only requirement for partition is that the projection of the lowest part of the molecule (i.e., the closest dot mass relatively to the plane of the pore) falls inside the pore. This means that, if the lowest part can enter inside the pore, then, the rest of the molecule is supposed to enter the pore by a suction effect; if not, the molecule will diffuse back to the bulk of the solution. After generating a high number of tests in each radial position, the distribution of the radial probability is obtained, and the partition coefficient, $\Phi$, is readily calculated by radial integration, using the same method of [10], according to:

$\Phi=2 \int_{0}^{1} p(\beta) \beta d \beta$

where $\beta$ is the radial dimensionless coordinate $\left(r / r_{p}\right)$, and $r_{p}$ is the pore radius. The results of the simulations are presented in Fig. 3a, by representing the obtained $\Phi$ as a function of $n_{k}$ for various values of $L / r_{p}$. Since the radius of gyration can be calculated from $n_{k}$ and $l_{k}$, the latter being obtained from $L$ and $n_{k}$, another way of representing the results of the simulations is by plotting $\ln (\Phi)$ as function of the quantity $\ln \left(\lambda_{h}\right)$, with $\lambda_{h}$ being defined as the ratio of $\left\langle h^{2}\right\rangle^{1 / 2}$ to the pore radius (see Fig. 3b). As it can be seen there is a clear correlation between $\ln \left(\lambda_{h}\right)$ and $\ln (\Phi)$. Since $\lambda_{h}$ can be related to $\lambda_{g}$ or $\lambda_{s}$ (respectively the ratios $r_{g} / r_{p}$ and $r_{s} / r_{p}$ ) by using of equations (4)-(7) one can relate $\Phi$ with $\lambda_{g}$ or $\lambda_{s}$ accordingly. To obtain a fitting curve for the values obtained from the simulations represented in Fig. $3 \mathrm{~b}$, software SPSS/TableCurve2D v.5.0 was used. This software provides a rank of the best fitting curves for a certain specified experimental data (two-dimensional data), from a large database of built-in functions. A cumulative SDS (symmetric double sigmoidal) function was found to be adequate for this purpose (equation (14)). A 

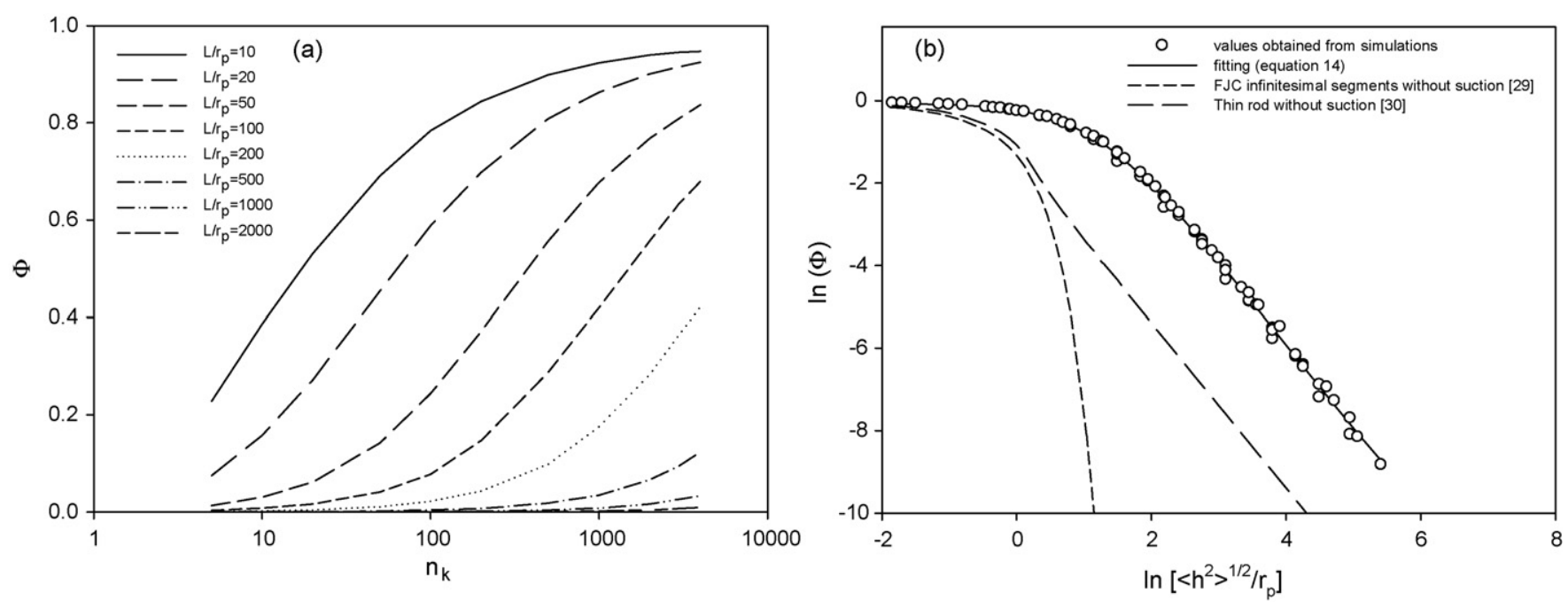

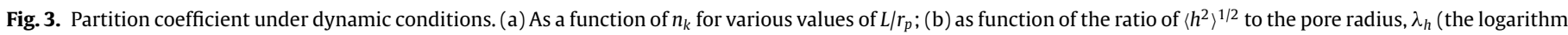
of both quantities is plotted).

squared correlation coefficient of 0.998 was obtained.

$$
\begin{aligned}
y= & a_{0}+\frac{a_{1}}{2 a_{3}}\left\{2 a_{4} \ln \left[\exp \left(\frac{x+\left(a_{3} / 2\right)}{a_{4}}\right)+\exp \left(\frac{a_{2}}{a_{4}}\right)\right]\right. \\
& \left.-2 a_{4} \ln \left[\exp \left(\frac{a_{2}+\left(a_{3} / 2\right)}{a_{4}}\right)+\exp \left(\frac{x}{a_{4}}\right)\right]+a_{3}\right\} \\
x= & \ln \left(\lambda_{h}\right) ; \quad y=\ln (\Phi) \\
a_{0}= & -11.60 ; \quad a_{1}=11.53 ; \quad a_{2}=3.955 ; \quad a_{3}=-5.520 ; \\
a_{4}= & -0.613
\end{aligned}
$$

Equation (14) can be compared with two limiting cases of permeation of linear molecules without the suction effect: that of a Gaussian chain with infinitesimally short segments [29], and the case of a thin rod [30]. Both references are cited by Davidson et al. [10]. Care should be taken when using equation 18a of reference [30] (see note in Appendix A). The comparison presented in Fig. 3b clearly shows the effect of suction on the partition coefficients.

A different approach was proposed previously [31], by assuming a different condition for the entrance of a flexible molecule into a pore, by considering the occurrence of a flow induced deformation, due to the velocity gradient at the entrance of a cylindrical pore. This gradient causes an elongational shear; then, it is assumed that the molecule follows perfectly the deformation induced by the flow, if that shear exceeds a certain critical value. If that value is reached at a distance $r_{c}$ from the pore entrance, then, for $r \leq r_{\mathrm{c}}$, the molecule is assumed to adopt a transversal dimension, presumably given by $R_{\perp}=\left\langle h^{2}\right\rangle^{1 / 2}\left(r / r_{\mathrm{c}}\right)$. The entrance condition is that $R_{\perp}\left(r=d_{p}\right)=d_{p}$, where $d_{p}$ is the pore diameter. This approach was further developed, in a recent study of permeation of supercoiled DNA through ultrafiltration membranes [8] aiming at the prediction of the critical flux, above which the permeation departs from nearly zero to significant values.

\subsection{Estimation of solute rejection}

After the estimation of the partition coefficients the next step in the set-up of the model is the estimation of intrinsic rejections. This can be a complex problem if $\lambda_{s}$ (or $\lambda_{h}$ ) values are small, though there are available relationships for $\lambda_{s}$ up to 0.87 . However, in the case of a flexible molecule much larger than the pore, the problem can be considerably simplified, since it is reasonable to assume that there will be a tendency for the molecule to almost completely fill the cross-sectional area of the pore. In physical terms, this corresponds to a situation in which diffusion inside the pore is severely hindered, and therefore, solute transport will be made exclusively by convection. When diffusion is absent, the solute molar (or mass) flux through the membrane is given by (see Appendix B):

$J=K_{c} c v$

where $v$ is the average velocity of the fluid inside the pore, $c$ is the concentration of the solute inside the pore, and $K_{c}$ is the hindrance factor for convection [1]. However, if the molecule occupies the entire cross-section of the pore, its velocity must be equal to the average velocity of the fluid, and therefore $K_{c} \rightarrow 1$. Moreover, since diffusion is negligible, the intrinsic rejection should be independent of the flux and given by [1] (see also Appendix B):

$R_{\infty}=1-\Phi K_{C}$

where $\Phi$ is the partition coefficient, and $R_{\infty}$ denotes the asymptotic value of the intrinsic rejection, which is independent of flux. Therefore, under these very special circumstances, i.e., only in the case of molecules with hydrodynamic radius (or radius of gyration) much larger than the pore radius, it is obtained that:

$R_{\infty}=1-\Phi$

Since this quantity is an intrinsic rejection, it can be related with the ratio of the concentration of the solute in the permeate, $C_{p}$, to the concentration near the membrane, $C_{m}$, according to:

$R_{m}=1-\frac{C_{p}}{C_{m}} \Rightarrow R_{\infty}=1-\left(\frac{C_{p}}{C_{m}}\right)_{\infty}$

where $R_{m}$ denotes intrinsic rejection. In order to estimate the observed rejection, which is defined as:

$R_{o b s}=1-\frac{C_{p}}{C_{b}}$

where $C_{b}$ is the concentration in the bulk of the feeding solution, the following relationship can be used, which is obtained from the film theory [32]:

$R_{o b s}=\frac{1}{1+\left(\left(1 / R_{m}\right)-1\right) \mathrm{e}^{\left(J_{v} / k\right)}}$

where $J_{v}$ is the permeate flux and $k$ is the concentration polarization mass transfer coefficient. The mass transfer coefficient can be estimated using a suitable correlation for estimation of the Sherwood number as function of the Reynolds and Schmidt numbers, 
or experimentally determined using the method described in the following section.

\section{Materials and methods}

Dextran T2000 was purchased from Pharmacia, now commercialized by Pharmacosmos. This dextran is less than $5 \%$ branched according to the manufacturer. Plasmid pUC19 was obtained by fermentation, isolated and purified accordingly with the method developed by [33]. Then, the obtained supercoiled plasmid was linearized by treatment with Smal enzyme, from Takara Bio Inc. The complete digestion was followed by agarose gel electrophoresis. After the treatment, the enzyme was removed by size exclusion chromatography, using Sephacryl HR-S100 from Amersham as gel, in a $1.5 \mathrm{~cm} \times 20 \mathrm{~cm}$ column, at $1 \mathrm{ml} / \mathrm{min}$ of flow rate and $25^{\circ} \mathrm{C}$. The mobile phase was Tris- $\mathrm{HCl}, 10 \mathrm{mM}$ buffer, at $\mathrm{pH}$ 8. Before filtering, $\mathrm{NaCl} 0.15 \mathrm{M}$ was added to the plasmid solutions, to avoid charge interactions with the membrane, since the plasmid is a negatively charged molecule. A similar solution was used in the case of the tests with dextran T2000. The concentrations of the solutes in the solutions tested were $1 \mathrm{~g} / \mathrm{l}$ in the case of the dextran and $0.02 \mathrm{~g} / \mathrm{l}$ in the case of DNA.

The filtration tests were performed in a $10 \mathrm{ml}$ stirred cell from Amicon, model 8010. The stirrer was set at the desired rotational speed (previously calibrated). The flux was carefully controlled by the use of a peristaltic pump placed after the membrane, thus, working by suction. The pump was an ISMATEC, model ISM444, which has 8 rotating cylinders; this ensures an almost non-pulsed flow. Using this pump and adequate tubing, very low values of flux can be imposed, which is essential to avoid excessive concentration polarization to occur; otherwise, it would be much more difficult to interpret the experimental results, especially taking into account the very low diffusivities of the molecules tested.

The membranes used were two "track-etched" polycarbonate (TEPC) from Sterlitech, USA, with pore radii of 15 and $40 \mathrm{~nm}$ (nominal values), a polyacrylonitrile membrane from Millipore, of $300 \mathrm{kDa}$ cut-off, XM300 and a polyvinylidene fluoride (PVDF) membrane from DSS/Alfa-Laval, of $100 \mathrm{kDa}$ cut-off, FS4OPP. Both the XM300 and FS4OPP membranes were previously characterized with reference solutes, in order to determine the pore radii; 10.5 and $4.1 \mathrm{~nm}$ were obtained, respectively. The method of characterization is described in [7], and the value for the PVDF membrane is from that reference.

For estimation of concentration polarization mass transfer coefficients, the following correlation, valid for the stirred cell used in this work can be used [34,35]:

$\frac{k r_{\text {cell }}}{D_{\infty}}=0.23\left(\frac{\varpi r_{\text {cell }}^{2} \rho}{\eta}\right)^{0.567}\left(\frac{\eta}{\rho D_{\infty}}\right)^{0.33}$

where $D_{\infty}$ is the diffusion coefficient, $\omega$ is the angular velocity $(\mathrm{rad} / \mathrm{s}), \rho$ is the density, $\eta$ is the viscosity, and $r_{\text {cell }}$ is the cell radius. To avoid the need of determination of viscosity and density of the solutions, it is assumed that:

$k=A \omega^{0.567}$

where " $A$ " is a constant for a particular solution; then, using equation (20) in the form:

$\ln \left(\frac{1-R_{o b s}}{R_{o b s}}\right)=\ln \left(\frac{1-R_{m}}{R_{m}}\right)+\frac{J_{v}}{k}$

the proportionality constant " $A$ " was obtained for dextran and plasmid solutions, by plotting $\ln \left[\left(1-R_{o b s}\right) / R_{o b s}\right]$ vs. $\omega^{-0.567}$.

The rejections of dextran T2000 (and of the reference solutes used for membrane characterization) were determined using a refractive index differential detector Shodex RI-71, by direct injection of the samples. The detector was previously calibrated for each solute. An excellent sensitivity and reproducibility is obtained by this method. The rejections of the plasmid were determined by measuring the absorbance at $260 \mathrm{~nm}$, using an Ultrospect 3000 spectrophotometer from Pharmacia Biotech. Each permeate sample was obtained from the filtration of $10 \mathrm{ml}$ of initial solution; the first $0.5 \mathrm{ml}$ of filtrate were colleted to a first eppendorf tube to be rejected, and then $1 \mathrm{ml}$ to be analyzed (thus a total permeate volume of $1.5 \mathrm{ml}$ was collected). The number of determinations performed for each set of operating conditions depended on the dispersion of the experimental results obtained. Repetitions (4-5 performed with new membranes) ensured average values of $R_{o b s}$ with a precision of \pm 0.1 , or higher, with $95 \%$ of confidence.

It should be noted that the membranes were selected according to simulations previously carried out, by estimation of $R_{m}$ using the model and estimation of the parameters in equation (21) in order to obtain $k$. However, only after the experimental determination of the constant " $A$ " in equation (22) satisfactory predictions of $R_{o b s}$ were obtained.

\section{Results and discussion}

The experimental values of the constant " $A$ " of equation (22) for calculation of the mass transfer coefficients of dextran T2000 and linear pUC19, were determined by varying the rotation speed in the stirred cell between 60 and $300 \mathrm{rpm}$; for the dextran solutions, $A=1.04 \times 10^{-7} \mathrm{~m} \mathrm{~s}^{-1}(\mathrm{rad} / \mathrm{s})^{-0.567}$, and for the plasmid $A=6.87 \times 10^{-8} \mathrm{~m} \mathrm{~s}^{-1}(\mathrm{rad} / \mathrm{s})^{-0.567}$. These values were then used for the estimations of $R_{o b s}$ from the values of $R_{m}$, according to equation (20). The estimate of $R_{m}$ values was made by equation (17), from the values of $\Phi$, obtained using equation (14).

In the case of the ultrafiltration membranes, FS40PP and XM300, the average pore size was determined from rejection measurements of reference solutes, as indicated in the previous section. In the case of track-etched polycarbonate membranes, membrane characterization was not necessary, since the information about pore size distribution is approximately known [36]; this information was used in the calculations, by assuming a continuous lognormal distribution for the pore size.

The results of the permeation tests for the dextran in the different membranes tested, at 60 and 100 rpm, are presented in Fig. 4; superimposed are also indicated the corresponding theoretical predictions. Although the results show some tendency towards an underestimation of the rejections, the fact is that the pore size for having intermediate rejections is reasonably well predicted. The very high rejections with FS4OPP membrane $\left(r_{p}=4.1 \mathrm{~nm}\right)$ at low stirring speeds and the low rejections in case of TEPC $0.08 \mu \mathrm{m}$ $\left(r_{p}=40 \mathrm{~nm}\right)$ membrane were verified. For the membranes with intermediate values of rejection, the results are better predicted for the highest stirring speed used in these tests $(100 \mathrm{rpm})$, but this may be simply due to the fact that rejections are being somewhat underestimated. In the case of the plasmid, the agreement between the experimental values and the model is greater than in the case of the dextran (Fig. 5). This might be related with the fact that the relevant physical properties, like the molecular weight, thus size, for the plasmid are better defined than for the dextran. In fact, dextrans always have considerably high polydispersities, and this has always some effect on the rejections. To check for that possibility, the effect of polydispersity in the case of membrane XM300 was simulated by considering dextran "T2000" a mixture of dextrans of molecular weights from 600 to $3400 \mathrm{kDa}$, log-normally distributed, this gives $M_{w}=2000 \mathrm{kDa}$ and $M_{n}=1550 \mathrm{kDa}$, and a polydispersity of 1.3 , which can be considered typical for linear dextrans according to the data presented in [37]. As it can be seen in Fig. 4, a possible effect of polydispersity seems to be not responsible for the observed differences. Another observation is that the rejections do 

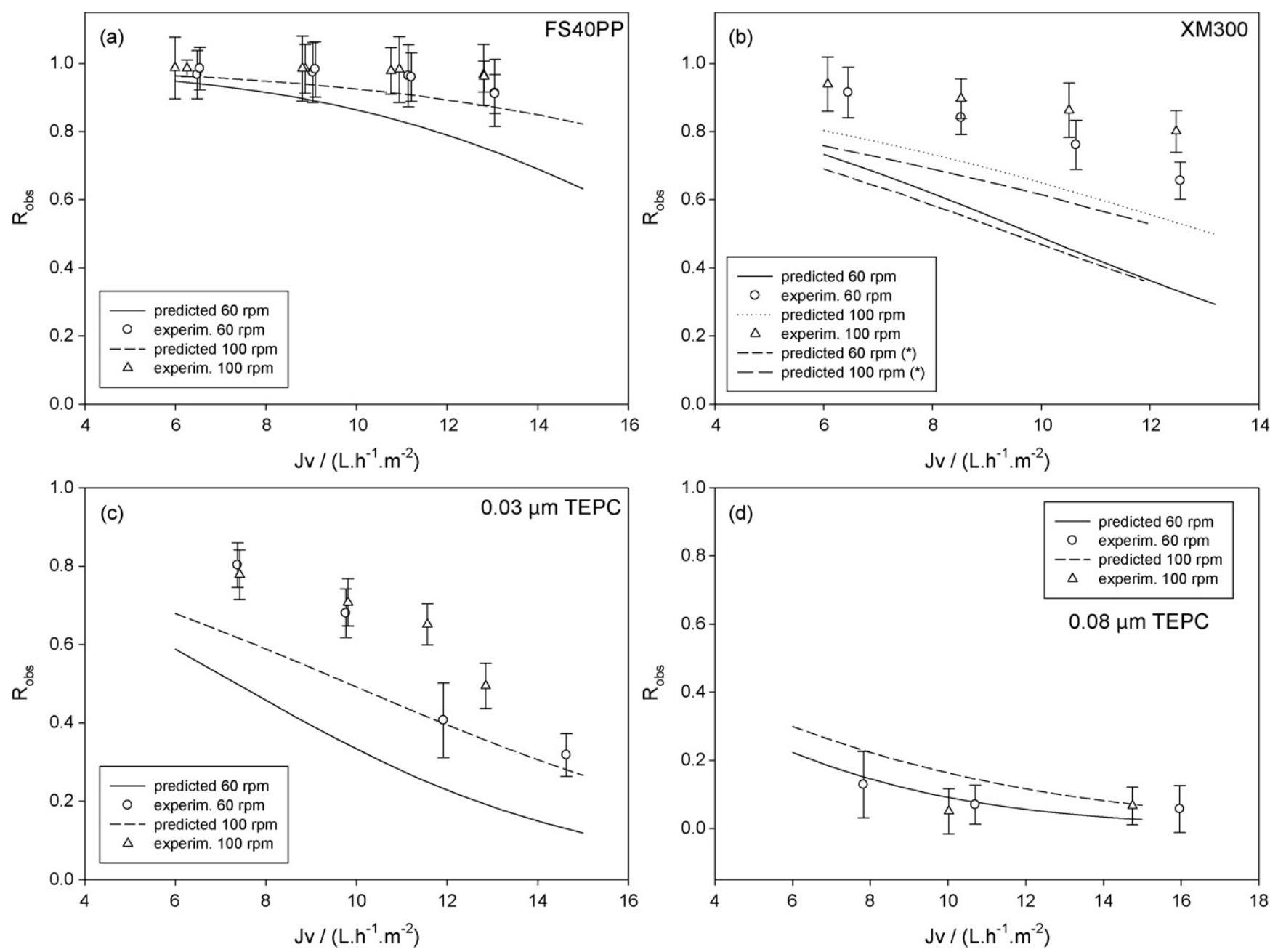

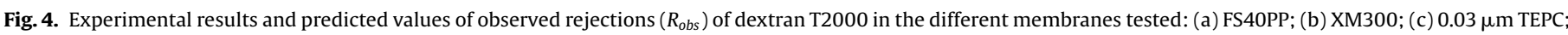
(d) $0.08 \mu \mathrm{m}$ TEPC. *Estimation considering polydispersity (see text for details).

not decrease as much as it was expected with the increase of the flux. This systematic tendency suggests that other effects that may influence rejections, especially at higher permeation fluxes, were not taken into account in the present approach. Nevertheless, from these obtained results, a clear conclusion can be made, that is the proposed model for the estimation of the rejections, in the case of very large macromolecules, is relatively accurate, thus confirming the idea that equation (17) can be used, with some degree of approach in these cases. The idea that convection is the dominating effect on the solute transport, is also confirmed, accordingly. This important result is also in accordance with those obtained by [38] in their study of transport of individual DNA molecules trough nanofluidic channels. In that work the authors determined the velocity of DNA molecules with 8.8, 20.3 and $48.5 \mathrm{kbp}$ through channels of comparable dimensions (in terms of order of magnitude; $175-3800 \mathrm{~nm}$ ) when subjected to a convective flow. The results are very clear in showing a linear dependence of the velocity of the molecules on the pressure gradient along the channel, and that for very narrow channels there is no dependence of the velocity on the molecular size; also, only above a certain channel height the differences in velocity appear, and then, for larger molecules higher velocities are observed. Therefore, it is not a surprise that in very narrow pores, such as those of membranes, the permeating molecules will occupy approximately the whole cross-section of the pores, and should move at a similar velocity as the fluid.
For a comparison of the results obtained here with the predictions of the deformation model previously cited in the introduction, one can calculate the values of critical flux, $J_{v, c}$ for significant permeation of the macromolecules studied. This can be adequately done by using the following expression adapted by [8] from the theory presented in [31]:

$J_{v, c}=\frac{\varepsilon k_{B} T}{r_{p}^{2} \eta}$

where $k_{B}$ is the Boltzmann constant, $T$ is the absolute temperature, $\eta$ is the fluid viscosity, and $r_{p}$ is the pore radius. As it can be seen from equation (24) the application of this model requires the knowledge of the membrane porosity, which is known from data provided by the manufacturer in the case of the TEPC membranes to be of 0.004 and 0.02 , respectively, for the membranes with nominal pore diameters of 0.03 and $0.08 \mu \mathrm{m}$. The approximate values of 280 and $190 \mathrm{~L} / \mathrm{h} \mathrm{m}^{2}$ are obtained for the 0.03 and $0.08 \mu \mathrm{m}$ membranes, respectively, using the nominal values of pore size. This clearly shows that both values of $J_{v, c}$ are significantly higher than the fluxes imposed in the experimental tests, and since permeation of both the dextran and the linearized plasmid was observed, one can conclude about the inadequacy of the deformation model to explain permeation of these molecules. An overestimation of the critical flux, using this model, was also concluded by Latulippe et al. [8] in the case of plasmid supercoiled DNA. 

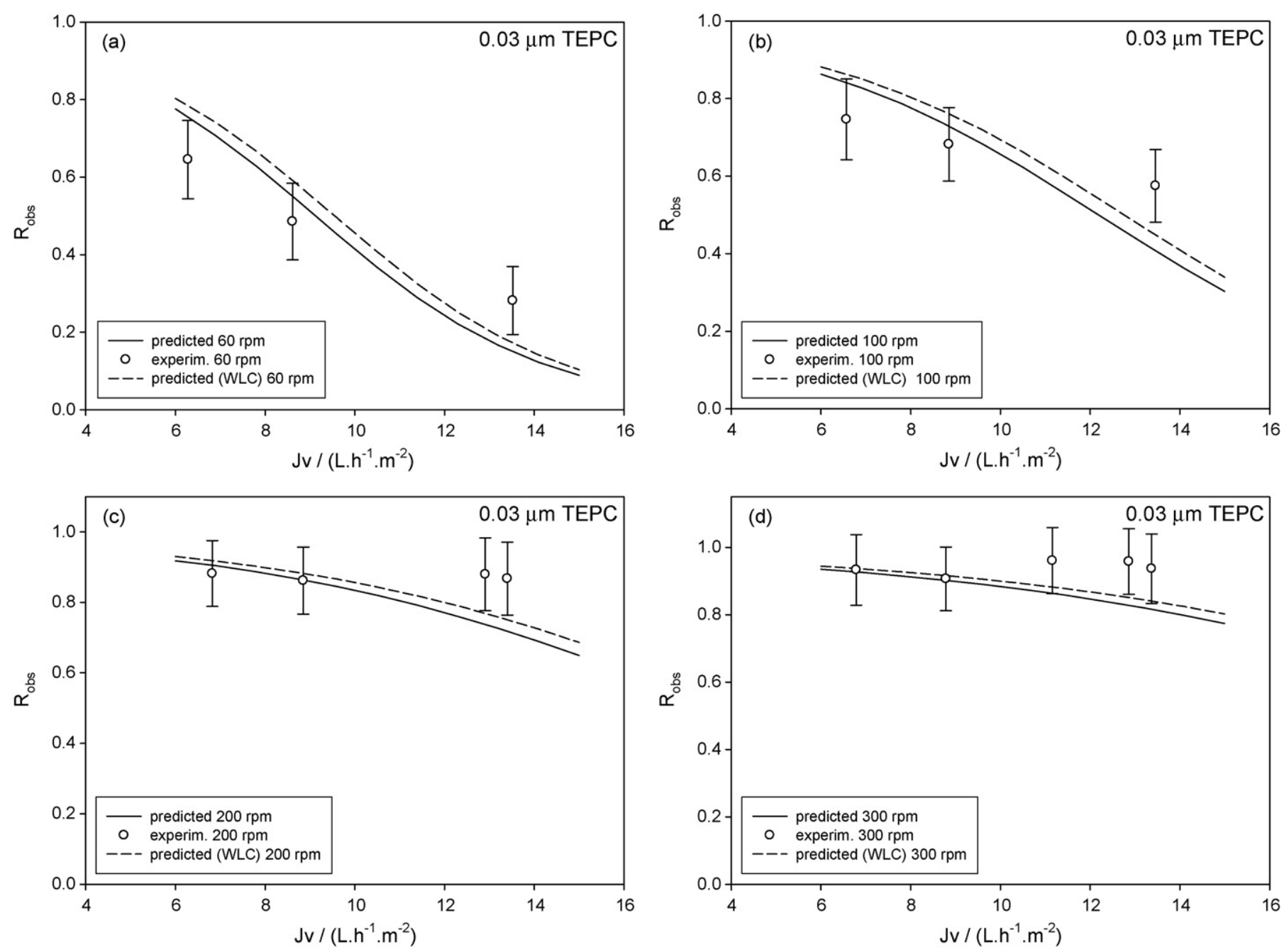

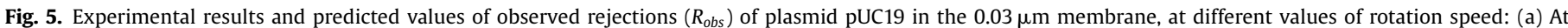

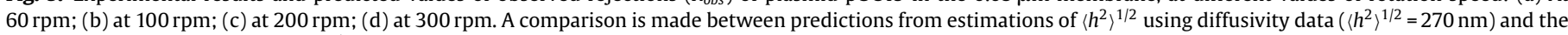
worm-like chain model $(\mathrm{WLC})\left(\left\langle h^{2}\right\rangle^{1 / 2}=294 \mathrm{~nm}\right)$.

\section{Conclusions}

A simple model that assumes the transport of a long molecule in narrow pores completely dominated by convection, was developed to predict the intrinsic rejections of two very large linear molecules, a dextran (T2000) and a linearized plasmid (pUC19) in different membranes. For the application of the model, partition coefficients were estimated using a stochastic method, in which it was assumed the suction of the molecule into the pore, if its lowest region falls inside the pore when the molecule approaches the membrane, aligned with the center of the pore, or aligned with 10 radial positions. For generating the molecular structure in the simulations, a freely jointed chain (FJC) model was used.

The partition coefficients were obtained as a function of the ratio of the root mean square end-to-end distance of the chain $\left(\left\langle h^{2}\right\rangle^{1 / 2}\right)$ to the pore radius. For the estimation of $\left\langle h^{2}\right\rangle^{1 / 2}$ the diffusion coefficients (or Stokes-Einstein radii) have to be known; in the case of linear plasmids a correlation was proposed from data collected from literature.

As predicted by the model, it was found that both molecules studied can permeate through pores of much smaller dimensions than the gyration radii and, actually, the predictions were found to be very accurate in the case of the plasmid. In the application of the model mass transfer coefficients of the solutes were calculated from the relationship $k=A \omega^{0.567}$, with the values of the parameter $A$ being experimentally determined for each solute. In the case of the dextran, the experimental results are generally higher than the model predictions, especially at the higher permeation fluxes. This same tendency can be observed also in the case of the plasmid, though the deviations are within the limits of the experimental error, in this case. Therefore, taking into account the simplicity of the proposed model, and the fact that it can be easily applied in practice, since it does not require parameters difficult to obtain in practice, its application can be considered successful. In future work, it will be important to understand the deviations observed for high fluxes, and to study the filtration of larger plasmids, in membranes with larger pores.

\section{Acknowledgement}

Fundação para a Ciência e a Tecnologia (FCT) for the grant awarded to António Morão (SFRH/BPD27170/2006). The authors thank Andreia Fonseca for helping in the experimental work.

\section{Appendix A}

A note should be made about equation (18a) of reference [30], since an error was detected. The correct form is: 
$K(\beta)=1-\frac{4}{3 \pi \beta}\left[\left(1+\beta^{2}\right) E\left(\frac{\pi}{2}, \beta\right)-\left(1+\beta^{2}\right) F\left(\frac{\pi}{2}, \beta\right)\right], \quad \beta \leq 1$

The symbols are those of [30].

\section{Appendix B}

When the dominant mechanism of solute transport is convection, its molar flux through the pores is given by [1,39]:

$J=W C_{m} v$

where $C_{m}$ is the solute concentration in the solution near the membrane, $v$ is the solvent velocity inside the pore and $W$ a convective hindrance factor, which [1] shows that corresponds to $\Phi K_{c}$. Therefore:

$J=\Phi K_{c} C_{m} v$

However, noting that $\Phi C_{m}=c$, where $c$ is the concentration inside the membrane, it follows that:

$J=K_{c} c v$

On the other hand, the solute flux through the pores, $J$, times the pore area, corresponds to $J_{v} C_{p} A_{m}$ where $J_{v}$ is the volumetric flux, defined per unit of membrane area and $A_{m}$ is the membrane area. Thus, taking into account that $v$ and $J_{v}$ are related through the membrane porosity, $\varepsilon$, and the pore area is given by $\varepsilon A_{m}$, it is obtained, by substituting in equation (B.2), that:

$$
\begin{aligned}
& \left(\frac{J_{v} C_{p}}{\varepsilon}\right)=\Phi K_{c} C_{m}\left(\frac{J_{v}}{\varepsilon}\right) \\
& \therefore \quad C_{p}=\Phi K_{c} C_{m} \Rightarrow R_{m} \equiv 1-\frac{C_{p}}{C_{m}}=1-\Phi K_{c}
\end{aligned}
$$

\begin{tabular}{|c|c|}
\hline \multicolumn{2}{|c|}{ Nomenclature } \\
\hline$a$ & persistence length $(\mathrm{m})$ \\
\hline$A$ & constant in equation $(22)\left(\mathrm{m} \mathrm{s}^{-1}(\mathrm{rad} / \mathrm{s})^{-0.567}\right)$ \\
\hline$A_{m}$ & membrane area $\left(\mathrm{m}^{2}\right)$ \\
\hline$c$ & $\begin{array}{l}\text { concentration of the solute inside the pore } \\
\left(\mathrm{mol} \mathrm{m}^{-3}\right)\end{array}$ \\
\hline$C_{m}$ & $\begin{array}{l}\text { concentration of the solute in the solution near the } \\
\text { membrane }\left(\mathrm{mol} \mathrm{m}^{-3}\right)\end{array}$ \\
\hline$C_{p}$ & $\begin{array}{l}\text { concentration of the solute in the permeate } \\
\left(\mathrm{mol} \mathrm{m}^{-3}\right)\end{array}$ \\
\hline$d_{p}$ & pore diameter $(\mathrm{m})$ \\
\hline & diffusion coefficient $\left(\mathrm{m}^{2} \mathrm{~s}^{-1}\right)$ \\
\hline$\left\langle h^{2}\right\rangle^{1 / 2}$ & average end-to-end distance of a polymer chain ( $\mathrm{m}$ ) \\
\hline$J$ & $\begin{array}{l}\begin{array}{l}\text { solute molar flux though the membrane } \\
\left(\mathrm{mol} \mathrm{s}^{-1} \mathrm{~m}^{-2}\right)\end{array}\end{array}$ \\
\hline$J_{v}$ & volumetric flux though the membrane $\left(\mathrm{m} \mathrm{s}^{-1}\right)$ \\
\hline$J_{v, c}$ & $\begin{array}{l}\text { critical volumetric flux though the membrane } \\
\left(\mathrm{m} \mathrm{s}^{-1}\right)\end{array}$ \\
\hline$k$ & mass transfer coefficient $\left(\mathrm{m} \mathrm{s}^{-1}\right)$ \\
\hline$k_{B}$ & Boltzmann constant $\left(8.314 \mathrm{~J} \mathrm{~mol}^{-1} \mathrm{~K}^{-1}\right)$ \\
\hline$K_{c}$ & hindrance factor for convection \\
\hline$l_{k}$ & Kuhn distance (m) \\
\hline$L$ & contour length of a polymer chain (m) \\
\hline$m$ & mass $(\mathrm{kg})$ \\
\hline$M_{n}$ & molecular weight, number average (Da) \\
\hline$M_{w}$ & molecular weight, mass average (Da) \\
\hline$n b p$ & number of base pairs \\
\hline$n_{k}$ & number of Kuhn segments \\
\hline$N$ & number of atoms in a molecule \\
\hline$r_{c}$ & $\begin{array}{l}\text { critical distance in Daoudi and Brochard [31] model } \\
(\mathrm{m})\end{array}$ \\
\hline
\end{tabular}

$r_{\text {cell }} \quad$ cell radius $(\mathrm{m})$

$r_{i}(i=0,1, \ldots, N-1)$ distance of an atom from the center of mass of a polymer chain (m)

$r_{g} \quad$ average gyration radius $(\mathrm{m})$

$r_{p} \quad$ pore radius $(\mathrm{m})$

$r_{s} \quad$ average hydrodynamic radius (m)

$r_{g}^{*} \quad$ instantaneous gyration radius $(\mathrm{m})$

$R_{m} \quad$ intrinsic rejection

$R_{\text {obs }} \quad$ observed rejection

$R_{\perp} \quad$ transversal dimension in Daoudi and Brochard [31] model ( $\mathrm{m}$ )

$R_{\infty} \quad$ intrinsic rejection at infinite flux

$T \quad$ absolute temperature (K)

$v \quad$ average velocity of the fluid inside the pore $\left(\mathrm{m} \mathrm{s}^{-1}\right)$

W convective hindrance factor

\section{Greek symbols}

$\varepsilon \quad$ membrane porosity

$\Phi \quad$ partition coefficient

$\eta \quad$ viscosity (Pa s)

$\lambda_{g} \quad$ the ratio of $\left\langle h^{2}\right\rangle^{1 / 2}$ to the gyration radius

$\lambda_{h} \quad$ the ratio of $\left\langle h^{2}\right\rangle^{1 / 2}$ to the pore radius

$\lambda_{s} \quad$ the ratio of $\left\langle h^{2}\right\rangle^{1 / 2}$ to the hydrodynamic radius

$\rho \quad$ density $\left(\mathrm{kg} \mathrm{m}^{-3}\right)$

$\omega \quad$ angular velocity $(\mathrm{rad} / \mathrm{s})$

\section{References}

[1] W.M. Deen, Hindered transport of large molecules in liquid-filled pores, AIChE J. 33 (1987) 1409-1425

[2] W.R. Bowen, J.S. Welfoot, Modelling the performance of membrane nanofiltration-critical assessment and model development, Chem. Eng. Sci. 57 (2002) 1121-1137.

[3] P. Dechadilok, W.M. Deen, Hindrance factors for diffusion and convection in pores, Ind. Eng. Chem. Res. 21 (2006) 6953-6959.

[4] A. Zydney, A. Xenopoulos, Improving dextran tests for ultrafiltration membranes: effect of device format, J. Membr. Sci. 291 (2007) 180-190.

[5] B. Van Der Bruggen, J. Schaep, D. Wilms, C. Vandecasteele, A comparison of models to describe the maximal retention of organic molecules in nanofiltration, Sep. Sci. Technol. 35 (2000) 169-182.

[6] T.R. Noordman, J.A. Wesselingh, Transport of large molecules through membranes with narrow pores: the Maxwell-Stefan description combined with hydrodynamic theory, J. Membr. Sci. 210 (2002) 227-243.

[7] A. Morão, M.T. Pessoa de Amorim, A. Lopes, I. Escobar, J.A. Queiroz, Characterisation of ultrafiltration and nanofiltration membranes from rejections of neutral reference solutes using a model of asymmetric pores, J. Membr. Sci. 319 (2008) 64-75.

[8] D.R. Latulippe, K. Ager, A.L. Zydney, Flux-dependent transmission of supercoiled plasmid DNA through ultrafiltration membranes, J. Membr. Sci. 294 (2007) 169-177.

[9] M.G. Davidson, W.M. Deen, Hindered diffusion of water-soluble macromolecules in membranes, Macromolecules 21 (1988) 3474-3481.

[10] M.G. Davidson, U.W. Suter, W.M. Deen, Equilibrium partitioning of flexible macromolecules between bulk solution and cylindrical pores, Macromolecules 20 (1987) 1141-1146.

[11] G.F. Hermsen, B.A. de Geeter, N.F.A. van der Vegt, M. Wessling, Monte Carlo simulation of partially confined flexible polymers, Macromolecules 35 (2002) 5267-5272.

[12] P. Cifra, T. Bleha, Simulations of sieving characteristics of macromolecules in porous membranes at high concentrations, J. Membr. Sci. 265 (2005) 51-59.

[13] A. Tager, Physical Chemistry of Polymers, MIR Publishers, Moscow, 1978.

[14] R.J. Young, P.A. Lovell, Introduction to Polymers, 2nd ed., CRC Press, 2000.

[15] I. Teraoka, Polymer Solutions: An Introduction to Physical Properties, Wiley Interscience, 2002.

[16] N.I. Abu-Lail, T.A. Camesano, Polysaccharide properties probed with atomic force microscopy, J. Microsc. 212 (2003) 217-238.

[17] S.F. Sun, Physical Chemistry of Macromolecules: Basic Principles and Issues, 2nd ed., Wiley Interscience, 2004.

[18] M. Fukatsu, M. Kurata, Hydrodynamic properties of flexible-ring macromolecules, J. Chem. Phys. 44 (1966) 4539-4545.

[19] R.M. Robertson, S. Laib, D.E. Smith, Diffusion of isolated DNA molecules: dependence on length and topology, Proc. Natl. Acad. Sci. U.S.A. 103 (2006) 73107314. 
[20] Y. Oono, M. Kohmoto, Renormalization group theory of transport properties of polymer solutions. I. Dilute solutions, J. Chem. Phys. 78 (1983) 520

[21] P.J. Hagerman, Flexibility of DNA, Ann. Rev. Biophys. Biophys. Chem. 17 (1988) 265-286.

[22] O. Kratky, G. Porod, Röntgenuntersuchung gelöster Fadenmoleküle, Rec. Trav. Chim. Pays-Bas. 68 (1949) 1106-1123.

[23] C. Bouchiat, M.D. Wang, J.-F. Allemand, T. Strick, S.M. Block, V. Croquette, Estimating the persistence length of a worm-like chain molecule from forceextension measurements, Biophys. J. 76 (1999) 409-413.

[24] D.E. Smith, T.T. Perkins, S. Chu, Dynamical scaling of DNA diffusion coefficients, Macromolecules 29 (1996) 1372-1373.

[25] G. Chirico, G. Baldini, Rotational diffusion and internal motions of circular DNA. I. Polarized photon correlation spectroscopy, J. Chem. Phys. 104 (1996) 6009-6019.

[26] S.S. Zakharova, W. Jesse, C. Backendorf, S.U. Egelhaaf, A. Lapp, J.R.C. van der Maarel, Dimensions of plectonemically supercoiled DNA, Biophys. J. 83 (2002) 1106-1118.

[27] T.A. Laurence, X. Kong, M. Jäger, S. Weiss, Probing structural heterogeneities and fluctuations of nucleic acids and denatured proteins, Proc. Natl. Acad. Sci. U.S.A. 102 (2005) 17348-17353.

[28] D. Voet, J. Voet, Biochemistry, 2nd ed., John Wiley \& Sons, 1995.

[29] E.F. Casassa, Equilibrium distribution of flexible polymer chains between a macroscopic solution phase and small voids, J. Polym. Sci., Polym. Lett. 5 (1967) 773-778.

[30] J.C. Giddings, E. Kucera, C.P. Russell, M.N. Myers, Statistical theory for the equilibrium distribution of rigid molecules in inert porous networks, J. Phys. Chem. 72 (1968) 4397-4408.

[31] S. Daoudi, F. Brochard, Flows of flexible polymer solutions in pores, Macromolecules 11 (1978) 751-758.

[32] T.K. Sherwood, P.L.T. Brian, R.E. Fisher, L. Dresner, Salt concentration at phase boundaries in desalination by reverse osmosis, Ind. Eng. Chem. Fundam. 4 (1965) 113-118.

[33] F. Sousa, S. Freitas, A.R. Azzoni, D.M.F. Prazeres, J. Queiroz, Selective purification of supercoiled plasmid DNA from clarified cell lysates with a single histidine-agarose chromatography step, Biotechnol. Appl. Biochem. 45 (2006) 131-140.
[34] W.S. Opong, A.L. Zydney, Diffusive and convective protein transport through asymmetric membranes, AIChE J. 37 (1991) 1497-1510.

[35] W.R. Bowen, A.W. Mohammad, N. Hilal, Characterization of nanofiltration membranes for predictive purposes-use of salts, uncharged solutes and atomic force microscopy, J. Membr. Sci. 126 (1997) 91-105.

[36] K.J. Kim, P.V. Stevens, Hydraulic and surface characteristics of membranes with parallel cylindrical pores, J. Membr. Sci. 123 (1997) 303-314.

[37] M.D. Lechner, D.G. Steinmeier, Sedimentation coefficients, diffusion coefficients, partial specific volumes, frictional ratios, and second virial coefficients of polymers in solution, in: J. Brandrup, E.H. Immergut (Eds.), Polymer Handbook, 3rd ed., Wiley Interscience, 1989.

[38] D. Stein, F.H.J. van der Heyden, W.J.A. Koopmans, C. Dekker, Pressure-driven transport of confined DNA polymers in fluidic channels, Proc. Natl. Acad. Sci. U.S.A. 103 (2006) 15853-15858.

[39] I. Teraoka, Polymer solutions in confining geometries, Prog. Polym. Sci. 21 (1996) 89-149.

[40] R. Lewis, R. Pecora, Comparison of predicted Rouse-Zimm dynamics with observations for a 2311 base pair DNA fragment, Macromolecules 19 (1986) 2074-2075.

[41] S.S. Sorlie, R. Pecora, A dynamic light scattering study of four DNA restriction fragments, Macromolecules 23 (1990) 487-497.

[42] G. Voordouw, Z. Kam, N. Borochov, H. Eisenberg, Isolation and physical studies of the intact supercoiled. The open circular and the linear forms of CoIE1-plasmid DNA, Biophys. Chem. 8 (1978) 171-189.

[43] J. Newman, J. Tracy, R. Pecora, Dynamic light scattering from monodisperse 2311 base pair circular DNA: ionic strength dependence, Macromolecules 27 (1994) 6808-6811.

[44] T.H. Nguyen, M. Elimelech, Plasmid DNA adsorption on silica: kinetics and conformational changes in monovalent and divalent salts, Biomacromolecules 8 (2007) 24-32

[45] S. Araki, T. Nakai, K. Hizume, K. Takeyasu, K. Yoshikawa, Hydrodynamic radius of circular DNA is larger than that of linear DNA, Chem. Phys. Lett. 418 (2006) 255-259.

[46] G.L. Lukacs, P. Haggie, O. Seksek, D. Lechardeur, N. Freedman, A.S. Verkman, Sizedependent DNA mobility in cytoplasm and nucleus, J. Biol. Chem. 275 (2000) 1625-1629. 